\title{
Médiévales
}

Langues, Textes, Histoire

48 | printemps 2005

Princes et princesses à la fin du Moyen Âge

\section{Princes et princesses à la fin du Moyen Âge}

\section{Didier Lett et Olivier Mattéoni}

\section{(2) OpenEdition}

Journals

Édition électronique

URL : https://journals.openedition.org/medievales/832

DOI : 10.4000/medievales.832

ISSN : 1777-5892

Éditeur

Presses universitaires de Vincennes

Édition imprimée

Date de publication : 1 juin 2005

Pagination : 5-14

ISBN : 2-84292-169-0

ISSN : 0751-2708

Référence électronique

Didier Lett et Olivier Mattéoni, «Princes et princesses à la fin du Moyen Âge », Médiévales [En ligne], 48 | printemps 2005, mis en ligne le 02 mars 2005, consulté le 22 avril 2022. URL : http://

journals.openedition.org/medievales/832; DOI : https://doi.org/10.4000/medievales.832

Ce document a été généré automatiquement le 22 avril 2022

Tous droits réservés 


\title{
Princes et princesses à la fin du Moyen Âge
}

\author{
Didier Lett et Olivier Mattéoni
}

1 De l'ouvrage de Michelle Bubenicek sur Yolande de Flandre ${ }^{1}$ au colloque du CRISIMA de 1999 dont l'objet était "Reines et princesses "2, en passant par les dernières biographies politiques sur les ducs et duchesses de Bourgogne (Charles le Téméraire, Philippe le Beau, Isabelle de Portugal ${ }^{3}$ ), par les récentes études consacrées aux reines ${ }^{4}$ ou le dossier des Annales HSS de 2002 intitulé «Du gouvernement des princes » 5 , l'historiographie française paraît aujourd'hui marquée par un fort engouement pour les princes et les princesses de la fin de l'époque médiévale ${ }^{6}$. Comment l'expliquer ? Par le retour du politique ${ }^{7}$ ? Par une attention soutenue portée depuis quelques années aux élites $^{8}$ ? Par la tradition française du genre monographique ? Par le nouvel intérêt pour la micro-histoire? Le retour des "grands personnages » sur la scène de l'Histoire ou, tout au moins, des hommes et des femmes appartenant aux familles et cercles « dirigeants ", serait-il un des symptômes de la « crise de l'Histoire »? Ou, au contraire, doit-il être interprété comme le signe d'un enrichissement? Le genre biographique, assumé par les plus grands médiévistes français - que l'on pense à Jacques Le Goff et son Saint Louis ${ }^{9}$, Bernard Guenée et ses Quatre vies de prélats français à la fin du Moyen $\hat{A} g e^{10}$, ou Françoise Autrand et ses Charles $V$ et Charles $V I^{11}$ - se présente désormais comme sujet d'histoire "globalisant ${ }^{12}$, intégrant les tendances nouvelles de la recherche : prosopographie ${ }^{13}$, histoire des représentations et de l'imaginaire, du droit, des «arts de gouverner ${ }^{14}$, des liens de parenté et d'«amitié» qui structurent la société politique.

2 À côté de ces multiples recherches centrées sur la personne du prince, de nombreux travaux ont vu le jour qui éclairent davantage un aspect particulier de l'activité princière: étude de l'entourage ${ }^{15}$, de l'action politique ${ }^{16}$, de l'œuvre monumentale, culturelle et "mémoriale $~^{17}$. Chaque fois, l'accent est mis sur une lecture politique au sens large du terme, qui intègre pratiques gouvernementales, considérations territoriales et réflexion idéologique ${ }^{18}$. 
3 Affectant les princes dans un premier temps ${ }^{19}$, ce mouvement gagne depuis quelques années les princesses et les reines ${ }^{20}$. Le regain d'intérêt pour ces dernières est directement lié au courant historiographique que représente l'histoire des femmes ${ }^{21} \mathrm{et}$, plus spécifiquement, l'histoire du pouvoir des femmes analysé selon une large perspective : commandement des abbesses ${ }^{22}$, pouvoir des épouses et des veuves ${ }^{23}$, autorité des femmes à l'intérieur de la sphère familiale ${ }^{24}$. Les femmes ainsi étudiées ont laissé plus que d'autres des traces dans l'Histoire. Éclairer leur parcours et leur action présente certes un danger, celui de renouer avec l'histoire des femmes d'exception, des "grandes figures $»^{25}$, écueil qui a été fort heureusement évité dans le large et salutaire mouvement de l'histoire des femmes en France ${ }^{26}$. Il n'empêche que l'étude du pouvoir exercé par les femmes doit avoir sa place au sein d'une histoire globale des pouvoirs, et il ne peut être analysé pour le Moyen Âge comme une « anomalie ». En outre, on fera remarquer que si l'abondante et récente littérature sur l'histoire des princesses en France est traversée par le courant historiographique hérité de l'histoire des femmes, elle reste cependant, même dans les études les plus récentes, assez peu sensible à l'histoire du genre ${ }^{27}$, outil de déconstruction et "démarche ${ }^{28}$ pourtant efficaces pour renouveler les approches et les perspectives de l'histoire du pouvoir et observer comment se construisent les catégories, permettant de poser un certain nombre de questions cruciales : quelle est la part du masculin ou du féminin dans l'art de régner, de gouverner, dans la tenue d'un conseil, dans une prise de décision? Qu'y a-t-il de « féminin » chez une princesse ? Son approche de la réalité politique, sa perception des individus, ses émotions sont-elles si différentes de celles du prince ? Doit-elle, comme l'écrit Christine de Pizan "avoir cœur d'homme " ${ }^{29}$ ? L'appartenance à un milieu princier joue-t-elle comme facteur de durcissement ou, au contraire, d'adoucissement des oppositions de genre? Faut-il d'abord apprendre à tenir son rang ou à «tenir son sexe $»^{30}$ ?

4 Les quatre articles ici rassemblés, rédigés par de jeunes chercheurs et chercheuses, illustrent parfaitement cet engouement et ces tendances historiographiques diverses. Deux d'entre eux nous offrent également à l'appui de très riches documents : des textes inédits, transcrits et traduits concernant Sforza Secondo, et une version intégrale modernisée de la Vie d'Isabelle de France par Agnès d'Harcourt, déjà publiée en 1668 par $\mathrm{Du}$ Cange. Les quatre contributions traitent de quelques figures de princes et de princesses laïques entre le milieu du XIII ${ }^{\mathrm{e}}$ siècle et le début du XVI ${ }^{\mathrm{e}}$ siècle, en Italie, en France ou en Allemagne. De leur lecture, trois thèmes majeurs de réflexion peuvent être retenus.

À la croisée des catégories

$5 \quad$ L'étude des princes et des princesses représente un poste d'observation privilégié pour élaborer une histoire des identités car ces "personnages" se situent aux confins de plusieurs catégories qu'il convient d'articuler entre elles: noblesse, jeunesse, sexe, aînesse. C'est de cette confrontation avec ces autres modes d'identification que sont le statut social ou juridique, l'âge, le sexe ou la position dans la parenté que l'historien peut parvenir à une meilleure approche de l'identité princière.

6 La plupart des hommes et des femmes éclairés ici, en effet, sont des jeunes, une catégorie fort difficile à cerner comme Georges Duby l'avait déjà montré dans son fameux article daté de $1964^{31}$. Les sociologues et les anthropologues, eux aussi, ces dernières années, ont beaucoup apporté à l'histoire des âges de la vie et de la jeunesse en tant que catégories construites offrant à l'historien un regard nouveau sur ce qu'on 
a pu longtemps considérer comme des "évidences naturelles ${ }^{32}$. Dans une situation donnée, laquelle de ces catégories, " jeunes ", « haute aristocratie », " sexe », domine ? Dans le cas de Sforza Secondo étudié par Pierre Savy, l'appartenance sociale semble primer le reste. Mais alors, comment faut-il définir la jeunesse dans le milieu princier? Les bornes chronologiques sont-elles plus précoces pour les filles du fait de leur âge au mariage?

7 À la fin du Moyen Âge, les notions de légitimité et de bâtardise sont également à prendre en compte dans les milieux princiers, même si, comme le montre Pierre Savy, le fait d'être un bâtard n'est pas un élément d'exclusion, bien au contraire : légitimé vers l'âge de 13 ans, Sforza Secondo reçoit une éducation de prince cadet que lui prodigue un précepteur. Néanmoins, son statut ne peut que le destiner au métier des armes. Ailleurs, la bâtardise princière ouvre les portes d'une carrière dans l'Église. Partout en tout cas, le $\mathrm{XV}^{\mathrm{e}}$ siècle voit, pour emprunter à Michael Hargsor, l'« essor des bâtards nobles $\aleph^{33}$ : si la bâtardise princière reste toujours facteur de hiérarchisation, elle n'est aucunement facteur d'exclusion.

Enfin, dans le cas particulier d'Isabelle de France, étudiée par Anne-Hélène Allirot, une autre catégorie interfère, celle de la sainteté qui, ici, n'est envisageable qu'en comparaison avec celle de son frère, saint Louis.

Éducation et formation

Comme le prouve l'ensemble des contributions, la formation et l'éducation des princes et des princesses apparaissent comme un enjeu majeur ${ }^{34}$. L'exemple de Sforza Secondo qui vient d'être évoqué l'a montré. Le cas d'Anne de France, duchesse de Bourbon, le confirme. Les enseignements qu'elle rédige à l'attention de sa fille Suzanne, sans doute entre 1503 et 1505, perpétuent la tradition des conseils aux princesses illustrée par Louis IX et les Enseignements à sa fille Isabelle, pendant féminin des Enseignements à son fils ${ }^{35}$. L'éducation des princes et des princesses passe donc par l'écriture, qui joue un rôle essentiel dans la production et la diffusion d'une idéologie morale et "sapientielle». Traités de pédagogie, "miroirs aux princes", mais aussi histoires et chroniques sont ainsi sollicités et prennent place au sein des bibliothèques princières comme autant de marqueurs qui disent tout à la fois un statut, un pouvoir et une espérance, celle de la sagesse princière, gage de bon gouvernement.

Bien sûr, les traités de pédagogie, les «miroirs aux princes » de la fin du Moyen Âge, s'inspirent toujours, peu ou prou, du modèle par excellence qu'est le De Regimine principum de Gilles de Rome. Toutefois, un autre texte a eu une influence loin d'être négligeable. Il s'agit du De eruditione filiorum nobilium de Vincent de Beauvais. L'un de ses intérêts est d'avoir été commandité par Marguerite de Provence en 1250. On le voit, dans cette production, le "féminin » n'est pas absent. Les princesses sont à la fois à l'origine de la rédaction de textes de sagesse, comme le montrent les figures d'Élisabeth de Bosnie, Gabrielle de Bourbon, Anne de France ou Suzanne de Bourbon, évoquées ou étudiées ici par Élodie Lequain, et destinatrices de cet enseignement - leurs filles ou les filles de la cour ${ }^{36}$.

11 Il semble que les traités d'éducation destinés aux princesses fassent peu de place à la perspective politique et soient surtout d'ordre moral. Le but est-il d'en faire des femmes de pouvoir ou des bonnes épouses, mères et veuves de prince ? Il y a là un point qu'il conviendrait d'approfondir pour dresser des tableaux parallèles des vices et des vertus des filles et des garçons. De même, des comparaisons entre ces traités destinés aux jeunes princes et princesses et ceux rédigés à l'intention de la petite et de la 
moyenne noblesse ne seraient pas inutiles ${ }^{37}$. La distinction de sexe se modifie-t-elle en fonction de la place occupée dans la hiérarchie sociale ? Quoi qu'il en soit, dans ces milieux princiers, un signe de la dissymétrie entre les sexes n'est guère contestable : les traités destinés aux garçons ne peuvent être écrits par une femme, alors que l'inverse n'est pas vrai.

L'écriture épistolaire ou celle d'un traité invite par ailleurs à réfléchir à l'autonomie de la plume féminine, en même temps qu'elle conduit à s'interroger sur la part réelle du féminin dans ces écrits faits souvent de collages, de réemplois, et de stéréotypes émanant du masculin. Les princesses éducatrices sont largement influencées par des hommes, hommes d'Église principalement - confesseurs, directeurs de conscience - qui «masculinisent » leurs propos, comme l'atteste l'action du dominicain Pierre Martin auprès d'Anne de France et de Suzanne de Bourbon. Dans l'ensemble des traités de princesses, les modèles historiques de bons gouvernants sont les mêmes que ceux qui traversent les miroirs destinés aux princes. Qu'il s'agisse de David, Salomon, Charlemagne ou encore Louis IX, ces références « fonctionnent » aussi pour les femmes comme des figures tutélaires.

13 Mais au-delà de cette sensibilité masculine et féminine, l'une des spécificités premières de ce groupe de l'aristocratie est de gouverner, et là se trouvent les enjeux de l'éducation et de l'apprentissage.

Pratique du pouvoir et entourage princier

Les contributions ici rassemblées se veulent un apport à une réflexion plus large sur la pratique du pouvoir, même si elles ne permettent pas toujours de répondre aux questions spécifiques que pose notamment le gouvernement des princesses : ainsi, ce dernier donne-t-il ou non une tonalité «féminine » au pouvoir princier? Le cas de la duchesse de Bourgogne, Isabelle de Portugal, tend à montrer que cette interrogation est loin d'être superflue. Si cette dernière a certes œuvré dans le même sens que son mari, Philippe le Bon, au renforcement des États bourguignons, elle a joué un rôle important en matière diplomatique en vue de promouvoir la paix ${ }^{38}$. Sans doute avonsnous là une particularité de la princesse de la fin du Moyen Âge qui ne revêt pas l'image d'une guerrière. La figure féminine de la princesse et de la reine est volontiers associée à la paix ${ }^{39}$. Froissart ne s'y trompe pas quand, décrivant la reddition de Calais, il explique que les Bourgeois n'ont eu droit à la vie sauve qu'à l'intervention ultime de la reine d'Angleterre, Philippa de Hainaut ${ }^{40}$.

La réflexion générale sur le pouvoir princier permet de poser la question de sa légitimation. Ainsi les princes maintiennent toujours un lien très étroit avec un «monastère dynastique » qui, par les prières, entretient la memoria de la dynastie, tel celui de Itzehoe pour le lignage Schauenburg, comtes de Holstein, étudié par Mathieu Olivier ${ }^{41}$. Cette légitimation passe également par la recherche de figures tutélaires, véritables modèles que proposent les traités (Charlemagne, saint Louis) ou par celle de racines romaines (princes du Holstein).

Enfin, ces quatre études permettent d'aborder un aspect central de la réalité du pouvoir princier, celui de son entourage. Si la composition, les spécificités des hôtels féminins, les principes et les modalités de délégation de pouvoir entre le prince et la princesse sont peu éclairés, en revanche, l'importance prise par certains individus dans l'ombre des hommes et des femmes de pouvoir apparaît nettement. L'une de ces figures est celle de l'« historien ", mise en valeur dans la contribution de Mathieu Olivier qui, étudiant le Chronicon Holtzatiae dû à la plume du Presbyter Bremensis, montre comment 
cet ouvrage historique a une finalité politique manifeste: permettre au prince de Holstein, le commanditaire, de se démarquer nettement des deux grandes entités politiques voisines que sont la Saxe et l'Empire. Ici l'histoire participe à la définition des pouvoirs et légitime l'autorité du prince, au prix d'une réécriture des événements tendant à effacer les ruptures dynastiques ${ }^{42}$.

Les textes réunis dans ce numéro de Médiévales ne prétendent pas, bien entendu, épuiser un sujet si riche. Ainsi disent-ils peu de choses du patronage des princes et des princesses, de leur rôle de fondateurs et fondatrices de monastères ou de monuments mémoriaux, de leurs fonctions de mécènes ou encore de commanditaires, sans qu'on sache toujours, à partir des actes, concernant la princesse $e^{43}$, ce qui relève de sa propre initiative ou de celle de son mari ou de sa parentée ${ }^{44}$. Ces quatre articles permettent avant tout d'attirer notre attention sur la manière dont un "vieux " sujet en plein chantier et en plein renouvellement est nécessairement "rajeuni » par des problématiques récentes. Aujourd'hui, étudier les princes et les princesses à la fin du Moyen Âge, c'est aussi contribuer à l'histoire des principautés, des élites, des cours, de la formation, de la parenté et des alliances, des transferts patrimoniaux, de la mise en place de la bureaucratie et de l'État moderne.

\section{NOTES}

1. M. Bubenicek, Quand les femmes gouvernent. Yolande de Flandre. Droit et politique au XIVe siècle, Paris, 2002 (Monuments et documents, 64).

2. Reines et princesses au Moyen Âge. Actes du cinquième colloque international de Montpellier, Université de Paul-Valéry (24-27 novembre 1999), Cahiers du CRISIMA, 5 (Publications de l'Université Paul-Valéry-Montpellier III), Montpellier, 2001.

3. H. Dubois, Charles le Téméraire, Paris, 2004, J.-M. Cauchies, Philippe le Beau, le dernier duc de Bourgogne, Turnhout, 2003 (Burgundica IV), M. Somme, Isabelle de Portugal, duchesse de Bourgogne. Une femme au pouvoir au XVe siècle, Villeneuve d'Ascq, 1998, dans l'attente d'une biographie consacrée à Jean sans Peur par Bertrand Schnerb, à paraître prochainement.

4. F. Cosandey, La Reine de France, Paris, 2000, qui porte sur la période moderne mais comporte un développement sur la fin du Moyen Âge.

5. Annales HSS, septembre-octobre 2002, p. 1164-1206. Ce dossier comprend deux articles : J. Pysiak, «Philippe Auguste. Un roi de la fin des temps? », p. 1165-1190, et J. Dakhlia, «Les Miroirs des princes islamiques : une modernité sourde ? », p. 1191-1206. À ce recensement qui ne prétend pas être exhaustif peut être ajouté : Les Princes et l'histoire du XIVe au XVIII siècle, Actes du colloque organisé par l'Université de Versailles-SaintQuentin et l'Institut Historique Allemand, Paris-Versailles, 13-16 mars 1996, dir. C. Grell, W. Paravicini et J. Voss, Bonn, 1998.

6. Ce mouvement affecte également la littérature anglo-saxonne comme en témoignent Isabel la Catolica, Queen of Castile : Critical essays, D. A. Boruchoff dir., New York, 2003, ou la série biographique de Richard Vaughan, Philipp the Bold ( $1^{\text {re }}$ éd. 1962, 
$2^{\mathrm{e}}$ éd. 1979), John the Fearless (1973), et Philip the Gold (1970), qui viennent de faire l'objet d'une réédition, Woodbridge, 2002.

7. Comme l'écrivent par exemple Bernard Guenée et Jean-François Sirinelli : « en une génération, l'histoire politique, délaissée, s'est imposée, triomphante », B. Guenée et J. Fr. Sirinelli, « L'Histoire politique » dans L'Histoire et le métier d'historien en France. 1945-1995, Fr. Bédarida dir., Paris, 1995, p. 301.

8. Les Élites urbaines au Moyen Âge, XXVII Congrès de la SHMESP (Rome, mai 1996), Paris, 1997 ; J.-Ph. Genet et G. Lottes dir., L'État moderne et les élites, XIII ${ }^{e}$-XVIII ${ }^{e}$ siècles. Apports et limites de la méthode prosopographique, Paris, 1996 ; pour l'époque carolingienne on peut citer R. Le Jan (dir.), La royauté et les élites dans l'Europe carolingienne au IX siècle, Lille, 1998, p. 383-408.

9. J. Le Goff, Saint Louis, Paris, 1996.

10. B. Guenée, Entre l'Église et l'État. Quatre vies de prélats français à la fin du Moyen Âge, Paris, 1987.

11. Fr. Autrand, Charles V, Paris, 1994 ; ID., Charles VI, Paris, 1986.

12. Rappelons ici les paroles de Jacques Le Goff dans l'introduction de son Saint Louis pour justifier son projet : « Habitué par ma formation d'historien à tenter une histoire globale, j'ai été rapidement frappé par l'exigence de la biographie à faire de son personnage ce que nous avons considéré, Pierre Toubert et moi, comme un sujet "globalisant" autour duquel s'organise tout le champ de la recherche. Or quel objet, plus et mieux qu'un personnage, cristallise autour de lui l'ensemble de son environnement et l'ensemble des domaines que découpe l'historien dans le champ du savoir historique ? Saint Louis participe à la fois de l'économique, du social, du politique, du religieux, du culturel ; il agit dans tous les domaines, en les pensant d'une façon que l'historien doit analyser et expliquer ». Mais plus loin, Le Goff montre toute la difficulté du genre pour échapper à l'« illusion biographique » dénoncée par Pierre Bourdieu ou à l'« utopie biographique » fustigée par Jean-Claude Passeron : Saint Louis, op. cit., p. 15-16 (pour la citation) et suivantes. Cette réflexion doit être rapprochée de celle développée par Bernard Guenée, pour lequel la biographie est « un genre difficile, voire impossible », Entre l'Église et l'État, op. cit., p. 13.

13. En témoigne le collectif À l'ombre du pouvoir. Les entourages princiers au Moyen Âge, études réunies par A. Marchandise et J.-L. Kupper, Genève, 2003, et J.-Ph. Genet et G. Lottes (dir.), L'État moderne et les élites..., op. cit.

14. Pour emprunter à $M$. Senellart, Les arts de gouverner. Du regimen médiéval au concept de gouvernement, Paris, 1995.

15. E. Gonzalez, Un prince en son Hôtel. Les serviteurs des ducs d'Orléans au $X V^{e}$ siècle, Paris, 2004.

16. Au sein d'une bibliographie importante, on peut citer : M. Jones, Ducal Brittany 1364-1399. Relations with England and France during the reign of Duke John IV, Oxford, 1970 (trad. fr. sous le titre La Bretagne ducale. Jean IV de Montfort (1364-1399) entre la France et l'Angleterre, Rennes, 1998) ; J. Kerhervé, L'État breton au XIV et XV siècles. Les ducs, l'argent et les hommes, 2 t., Paris, 1987 ; G. Castelnuovo, Ufficiali e gentiluomini. La società politica sabauda nel tardo medioevo, Turin, 1994 ; O. Mattéoni, Servir le prince. Les officiers des ducs de Bourbon à la fin du Moyen Âge (1356-1523), Paris, 1998 ; A. Barbero, Il ducato di Savoia. Amministrazione e corte di uno stato franco-italiano, Rome-Bari, 2002.

17. J.-Y. Copy, Art, société et politique au temps des ducs de Bretagne. Les gisants hautbretons, Paris, 1986, et C. Prigent, Pouvoir ducal, religion et production artistique en Basse- 
Bretagne, 1350-1575, Paris, 1992, et, plus récemment, par J.-M. Guillouët, Les portails de la cathédrale de Nantes. Un grand programme sculpté du XVe siècle et son public, Rennes, 2003. 18. Un bel exemple de cette approche multiple est fourni par les actes du colloque de Ripaille-Lausanne d'octobre 1990, Amédée VIII-Félix V, premier duc de Savoie et pape (1383-1451), éd. B. Andenmatten et A. Paravicini-Bagliani, Lausanne, 1992.

19. En témoigne le déjà ancien colloque de la Société des Historiens Médiévistes de l'Enseignement Supérieur Public de 1992 tenu à Brest, Les Princes et le pouvoir au Moyen Âge, XXIII ${ }^{e}$ Congrès de la SHMESP (Brest, mai 1992), Paris, 1993.

20. Concernant l'histoire des reines, citons parmi les travaux récents et les plus marquants : F. Cosandey, La Reine de France..., op. cit., ou Queens and Queenship in Medieval Europe, Londres, 2002 ; H. H. Maurer, Margaret of Anjou. Queenship and Power in Late Medieval England, op. cit. ; et pour les reines « capétiennes », C. WoLL, Die Königinnen des hochmittelalterlichen Frankreichs, 987-1237/1238, Stuttgart, 2002.

21. L'ouvrage de M. Bubenicek, Quand les femmes gouvernent..., op. cit., est révélateur de la volonté d'articuler histoire politique et histoire des femmes.

22. M. Parisse, Les nonnes au Moyen Âge, Le Puy, 1983.

23. On peut citer dans cette perspective, Women and Power in the Middle Ages, éd.

M. Erler et M. Kowalewski, Athens (Georgie)-Londres, 1988. Pour le haut Moyen Âge, voir M. Parisse dir., Veuves et veuvages au haut Moyen Âge, Paris, 1993.

24. Voir, en dernier lieu, J. A. McNAMARA, « Women and Power through the Family Revisited ", dans Gendering the master narrative, éd. M. C. Erler et M. Kowalewski, Ithaca, 2003, p. 17-30.

25. M. Bubenicek, Quand les femmes gouvernent..., op. cit., p. 14, écrit : « à sources exceptionnelles, personnage exceptionnel ».

26. Il suffit de lire l'introduction de Christiane Klapisch-Zuber au Tome II de l'Histoire des femmes en Occident, M. Perrot et G. Duby dir., Paris, 1991 (éd. italienne 1990), Le Moyen Âge, Chr. Klapisch-Zuber dir., p. 11-23.

27. Ces travaux méconnaissent, voire rejettent, le genre vu par M. Bubenicek comme une « conception anglo-saxonne dont on a pu s'apercevoir qu'elle avait quelque peu tendance à chercher un reflet des problèmes actuels dans une société très différente » : Quand les femmes gouvernent..., op. cit., p. 17, note 47. Le contraste est, bien entendu, saisissant avec la situation outre-Atlantique et outre-Manche. Citons uniquement P. Richards et J. Munns dir., Gender, Power, Privilege in Early Modern Europe, London, 2003. 28. Voir en dernier lieu sur cette conception du genre, I. Brian, D. Lett, V. Sébillotte Cuchet et G. Verdo, «Le Genre comme démarche », dans Hypothèses 2004, Travaux de l'École doctorale d'Histoire de Paris 1-Panthéon-Sorbonne, 2005, p. 277-295.

29. C. de Pizan, Le livre des trois vertus, BnF, ms. fr. 452, fol. $61 \mathrm{v}^{\circ}$, au sujet de la «baronnesse »; cité par M.-Th. Caron, « Mariage et mésalliance : la difficulté d'être femme dans la société nobiliaire à la fin du Moyen Âge ", dans La femme au Moyen Âge. Actes du colloque de Maubeuge d'octobre 1988, M. Rouche et J. Heuclin éd., Maubeuge, 1990, p. 315.

30. Cordula Nolte, étudiant les électeurs et margraves du Brandebourg-Ansbach vers 1500, montre ainsi ce que la gender history apporte à l'étude des dynasties princières. En particulier, en travaillant sur les directions prises par les correspondances à l'intérieur des familles, elle dévoile que le genre, à côté de l'âge et de la génération, est un critère de distinction fondamental; cf. C. Nolte, « Gendering Princely Dynasties : Some Notes on Family Structure, Social Networks, and Communication at the Courts of the 
Margraves of Brandebourg-Ansbach around 1500 » dans Gendering in the Middle Ages, P. Stafford et A. B. Mulder-Bakker dir., Malden (Massachusetts), 2001, p. 174-191. 31. G. Duby, « Les “jeunes” dans la société aristocratique dans la France du Nord-Ouest au XII ${ }^{\mathrm{e}}$ siècle ", Annales ESC, 1964, p. 835-846, repris dans Id., Hommes et structures du Moyen Âge, Paris, 1979, et dans Id., La société chevaleresque, Paris, 1988, p. 129-142.

32. Sur la jeunesse comme catégorie aux frontières indécises, on renverra rapidement, pour la sociologie, à l'article devenu désormais classique de Pierre Bourdieu, P. Bourdieu, « La “jeunesse” n'est qu'un mot », Questions de sociologie, Paris, 1980, $2^{\mathrm{e}}$ éd. Paris, 1984, p. 143-154 et à L. Thévenot, « Une jeunesse difficile : les fonctions sociales $\mathrm{du}$ flou et de la rigueur dans les classements ", Actes de la Recherche en Sciences Sociales, 26-27, 1979, p. 3-18. En anthropologie, on peut utilement se référer à Passages à l'âge d'homme, L'Homme, 167-168, juillet-décembre 2003.

33. Article pionnier que cet article de M. Hargsor, « L'essor des bâtards nobles au $\mathrm{XV}^{\mathrm{e}}$ siècle ", Revue historique, 253, 1975, p. 319-354. Pour une étude récente sur un prince bâtard, voir A. de Riedmatten, Humbert le Bâtard. Un prince aux marches de la Savoie, Lausanne, 2004 (Cahiers lausannois d'Histoire médiévale 35).

34. Un exemple récent, offrant l'avantage de livrer des sources, est fourni par N. Blancardi, Les Petits princes. Enfance noble à la cour de Savoie (XVe siècle), Lausanne, 2001 (Cahiers lausannois d'Histoire médiévale 28).

35. On notera que Charles V avait offert à son beau-frère, le duc Louis II de Bourbon, un exemplaire des Enseignements de Louis IX à son fils et un autre exemplaire des Enseignements de Louis IX à sa fille: H. Delaborde, « Le texte primitif des Enseignements de saint Louis à son fils ", Bibliothèque de l'École des Chartes, 73, 1912, p. 85-91.

36. Pour l'éducation des rois et des princes anglais, voir N. Orme, From Childhood to Chilvary. The Education of the English Kings and Aristocracy 1066-1530, Londres, 1984.

37. L'exemple le plus connu de traité adressé à des jeunes filles au sein d'une moyenne noblesse est celui du Chevalier de la Tour Landry rédigé vers 1371, Le Livre du Chevalier de La Tour Landry pour l'Enseignement de ses filles, A. de Montaiglon éd., Paris, 1854.

38. M. Sommé, Isabelle de Portugal, duchesse de Bourgogne..., op. cit.

39. Voir N. Offenstadt, «Les femmes et la paix à la fin du Moyen Âge : genre, discours, rites ", dans Le Règlement des conflits au Moyen Âge, XXXI Congrès de la SHMESP (Angers, juin 2000), Paris, 2001.

40. J.-M. Moeglin, Les bourgeois de Calais. Essai sur un mythe historique, Paris, 2002, p. 40-41.

41. Même constat pour les ducs Valois de Bourgogne avec la chartreuse de Champmol, ou pour les ducs de Bourbon avec l'église prieurale de Souvigny.

42. Tout cela est à replacer dans le contexte plus large des rapports entre histoire et pouvoirs avec l'ouvrage classique de B. Guenée, Histoire et culture historique dans l'Occident médiéval, Paris, 1980.

43. Il faut constater que bien des actes de princesses attendent leur éditeur. Saluons ici le travail de M. Jones, Recueil des actes de Charles de Blois et Jeanne de Penthièvre, duc et duchesse de Bretagne (1341-1364), suivi des actes de Jeanne de Penthièvre (1364-1384), Rennes, 1996.

44. L. L. Gee, Women, Art and Patronage from Henry III to Edward III : 1216-1377, Woodbridge, 2002 qui montre, entre autres, que les femmes de sang royal ou issues de la haute noblesse ont joué un rôle essentiel dans l'évolution des goûts artistiques, en particulier dans l'importation des goûts continentaux en Angleterre, à cause des liens 
étroits (familiaux) qu'elles entretenaient avec les familles royales françaises (chapitre VII).

AUTEURS

OLIVIER MATTÉONI

Université Paris 1-Panthéon-Sorbonne, UFR d'Histoire, 17, rue de la Sorbonne, F-75005 Paris 